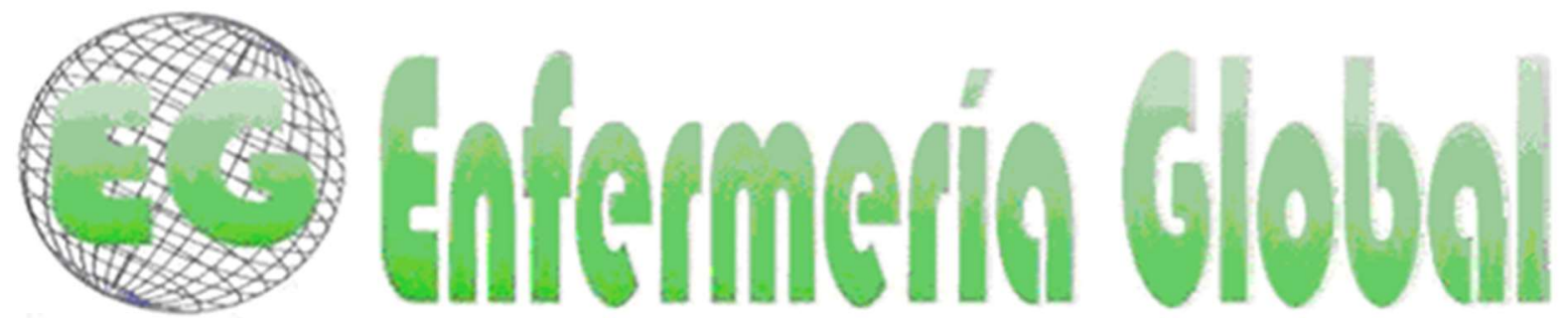

\title{
REVISIONES
}

\section{Las poblaciones vulnerables enfrentando los desafíos durante la pandemia del covid-19: una revisión sistemática}

Vulnerable populations' coping in facing challenges during the covid-19 pandemic: a systematic review

\author{
Lina Anisa Nasution ${ }^{1}$ \\ Anung Ahadi Pradana ${ }^{2}$ \\ Casman $^{3}$
}

1 Departamento de Enfermería, Universidad Pendidikan Indonesia, Bandung, West Java, 40154, Indonesia. linaanisa@upi.edu

${ }^{2}$ Programa de Estudio de Enfermería, Sekolah Tinggi IImu Kesehatan Mitra Keluarga. Indonesia.

${ }^{3}$ Departamento de Enfermería de Salud Infantil, Sekolah Tinggi IImu Kesehatan RS Husada. Indonesia.

\section{https://doi.org/10.6018/eglobal.456301}

Recibido: 16/11/2020

Aceptado: 3/03/2021

\section{RESUMEN:}

Objetivo: Durante la pandemia, la aplicación del distanciamiento social determina que las poblaciones vulnerables se conviertan en uno de los grupos que experimentan los peores impactos, especialmente niños, mujeres embarazadas y adultos mayores. El propósito de este estudio es analizar los obstáculos que experimentan las poblaciones vulnerables.

Método: Este estudio utiliza un método de búsqueda de literatura en varias bases de datos como CINAHL, Proquest, Wiley, ScienceDirect de 2015-2020.

Resultados: Los resultados de la búsqueda obtuvieron 17 artículos. La revisión crítica que se llevó a cabo siguió las directrices del Programa de Habilidades de Evaluación Crítica (CASP) de Cochrane. Los resultados de este estudio fueron las condiciones pandémicas que ocurren durante un largo período y pueden afectar a poblaciones vulnerables de la sociedad, que consisten en niños, mujeres embarazadas y adultos mayores. Las poblaciones vulnerables tienen varios mecanismos de supervivencia y están significativamente influenciadas por la presencia o ausencia de apoyo social de la familia, la comunidad y el gobierno.

Conclusiones: La importancia del papel del gobierno en prestar atención a la calidad de los servicios especiales de salud para poblaciones vulnerables puede mejorar el bienestar y el estado de salud de estas poblaciones.

Palabras clave: afrontamiento, COVID-19, pandemia, poblaciones vulnerables.

\section{ABSTRACT:}

Primary Goal: During the pandemic, the application of social distancing resulting in vulnerable populations becoming one of the groups experiencing the worst impacts, especially those who are children, pregnant women, and older adults. The purpose of this study is to analyze the obstacles encountered by these vulnerable populations. 
Methods: This study uses a literature search method on several databases, namely CINAHL, Proquest, Wiley, and ScienceDirect, in the 2015-2020 period.

Results: The search resulted in 17 articles. The critical review carried out followed the Critical Appraisal Skills Program guidelines from Cochrane. This study's results were pandemic conditions that occur over a long period can affect vulnerable populations in society, consisting of children, pregnant women, and older adults. Vulnerable people have various coping mechanisms and are significantly affected by the presence or absence of social support from family, community, and the Government.

Conclusions: The importance of the Government's role in paying attention to the quality of specific health services for vulnerable populations can improve these populations' welfare and health status.

Key words: coping, COVID-19, pandemic, vulnerable populations.

\section{INTRODUCCIÓN}

EI COVID-19 en 2020 afecta a 216 países, causando 17,660,523 casos confirmados y 680,894 muertes en el mundo(1). Los últimos datos infográficos de Indonesia mostraron que el número de pacientes con COVID-19 a mediados de septiembre de 2020 alcanzó los 320.564 casos con una cifra de muertos de 11.580 personas $^{(2)}$. El gobierno ha realizado varios esfuerzos, incluido el examen y tratamiento de los enfermos, ejecutando diversas promociones de salud relacionadas con la prevención de la transmisión. Las medidas preventivas recomendadas son buenas prácticas para el lavado de manos, equipo de protección personal, políticas regionales de cuarentena (cierre) y restricciones sociales a gran escala( ${ }^{(3,4)}$. El impacto de COVID-19 ha afectado la vida de las personas, varios sectores del desarrollo del país y la economía global( ${ }^{(4,5)}$.

La política de Adaptación de Nuevos Hábitos avanza, con la evaluación continua de la tasa de reproducción de COVID-19 y su impacto en todos los niveles de la sociedad. Los agregados que se centran en esta pandemia son las poblaciones vulnerables que abarcan las condiciones de salud y socioeconómicas ${ }^{(6)}$. Las condiciones de pandemia y desastre generalmente tienen un impacto significativo en la vida de las personas, especialmente aquellas que caen dentro de las poblaciones vulnerables. Las poblaciones vulnerables son grupos de personas que experimentan dificultades 0 incapacidad para anticipar y llevar a cabo el proceso de recuperación cuando se ven afectados por un desastre. Los que se encuentran en comunidades vulnerables son adultos mayores, personas con enfermedades no transmisibles, niños y mujeres embarazadas ${ }^{(7)}$.

El número de casos nuevos está aumentando hasta la fecha en Indonesia. El Ministerio de Salud de la República de Indonesia publicó que el número de casos confirmados aumenta en más de 3000 casos cada día en 2020. El creciente número de casos de COVID-19 ha llevado al Gobierno a desarrollar varias pautas de manejo y prevención, especialmente para los grupos vulnerables. Los procedimientos disponibles incluyen (a) para mujeres embarazadas, madres postparto y bajo peso al nacer durante el distanciamiento social, (b) para apoyo de salud mental y psicosocial, (c) para servicios de salud para niños pequeños, (d) para protección de las personas mayores con perspectiva de género durante el período COVID-19.

Una encuesta de 6854 encuestados sobre COVID-19 mostró que solo el $2 \%$ de ellos declaró que había sido diagnosticado y el $6 \%$ indicó que sabía que alguien estaba infectado. Los encuestados más jóvenes desconfían de COVID-19. El afrontamiento que llevan a cabo los individuos frente al síndrome de estrés correspondiente durante 
el aislamiento independiente incluye ver televisión o películas, limpiar la casa, mantener la comunicación con otras personas y pensar que el aislamiento es fundamental para ayudar a la comunidad ${ }^{(8)}$. Durante la pandemia, todos los cambios requieren la recopilación de datos sobre los factores de riesgo para reducir la gravedad de este desastre en particular para enfrentar una nueva vida lo antes posible ${ }^{(9)}$. En otras palabras, la comunidad debe poder adaptarse y afrontarlo bien. El concepto de adaptación asume que alguien es un sistema abierto influenciado por estímulos internos y externos. Los estímulos ambientales se componen de focales, contextuales y residuals ${ }^{(10)}$. El modelo de adaptación de Roy es un método eficaz para controlar problemas físicos y psicológicos y lograr que alguien se adapte al estímulo recibido(11).

El propósito de este trabajo es analizar los obstáculos experimentados por las poblaciones vulnerables para que algunos esfuerzos puedan eliminar las barreras como una forma de afrontarlas. Estos hallazgos serían un insumo para que las personas vulnerables se adapten a los obstáculos durante una pandemia.

\section{MÉTODOS}

Este estudio utiliza un método de búsqueda bibliográfica en varias bases de datos, a saber, CINAHL, Proquest, Wiley, ScienceDirect y utiliza palabras clave: "pandemia", "niños", "mujeres embarazadas", "adultos mayores", "poblaciones vulnerables" y "afrontamiento" de 2015 - 2020. Los resultados fueron 1277 artículos que coinciden con las palabras clave, seleccionaron la cita gemela y dieron como resultado 286 artículos. Se aplicaron criterios de exclusión e inclusión, en otros 17 artículos. La Figura 1 ilustra el proceso de búsqueda y selección de artículos. Los criterios de inclusión incluyen (a) artículos en idioma inglés, (b) el período de publicación 20152020, (c) diseño de estudio ECA, metanálisis, cohortes, encuestas, informes de casos y, (d) artículos sobre cómo hacer frente a las poblaciones vulnerables durante una pandemia.

Figura 1 El diagrama PRISMA en la obtención de los artículos relevantes

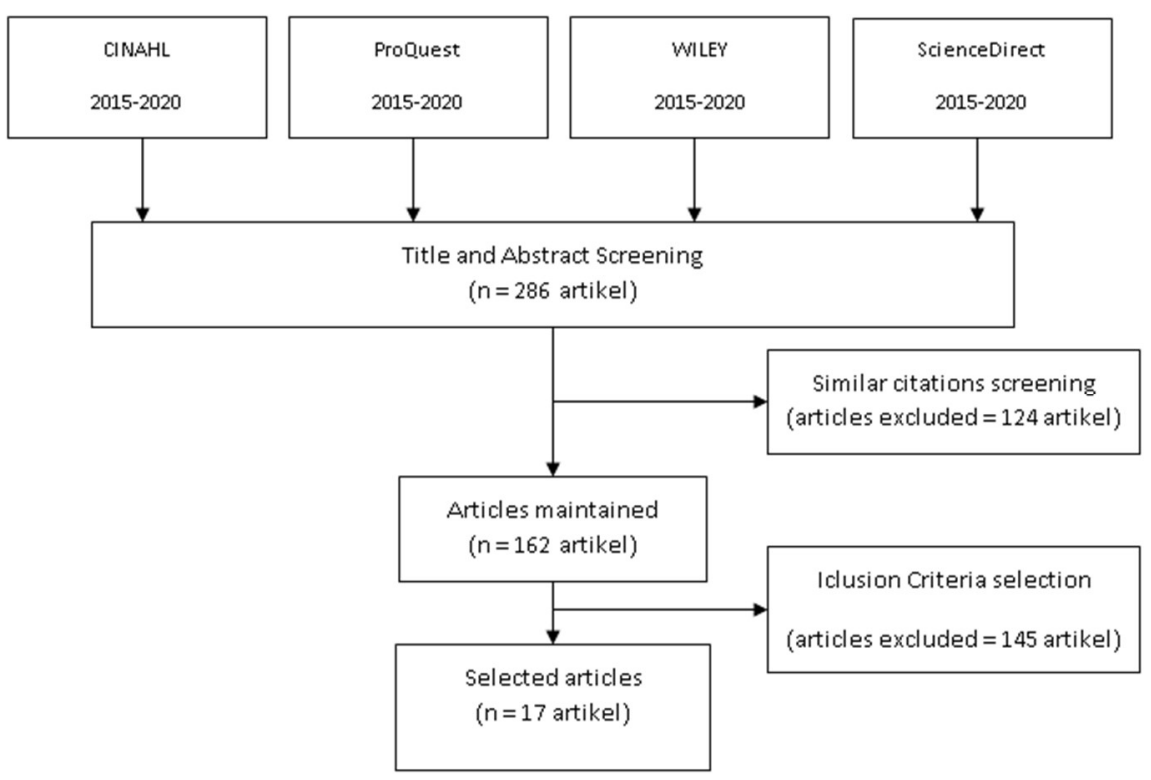




\section{RESULTADOS}

La revisión crítica siguió las pautas del Programa de Habilidades de Evaluación Crítica (CASP) de Cochrane; Se analizaron 17 artículos seleccionados utilizando los medios de seguimiento de riesgo de sesgo de RobVis para obtener los resultados de la extracción de riesgo de sesgo (Figura 2).

Figura 2 Riesgo de sesgo

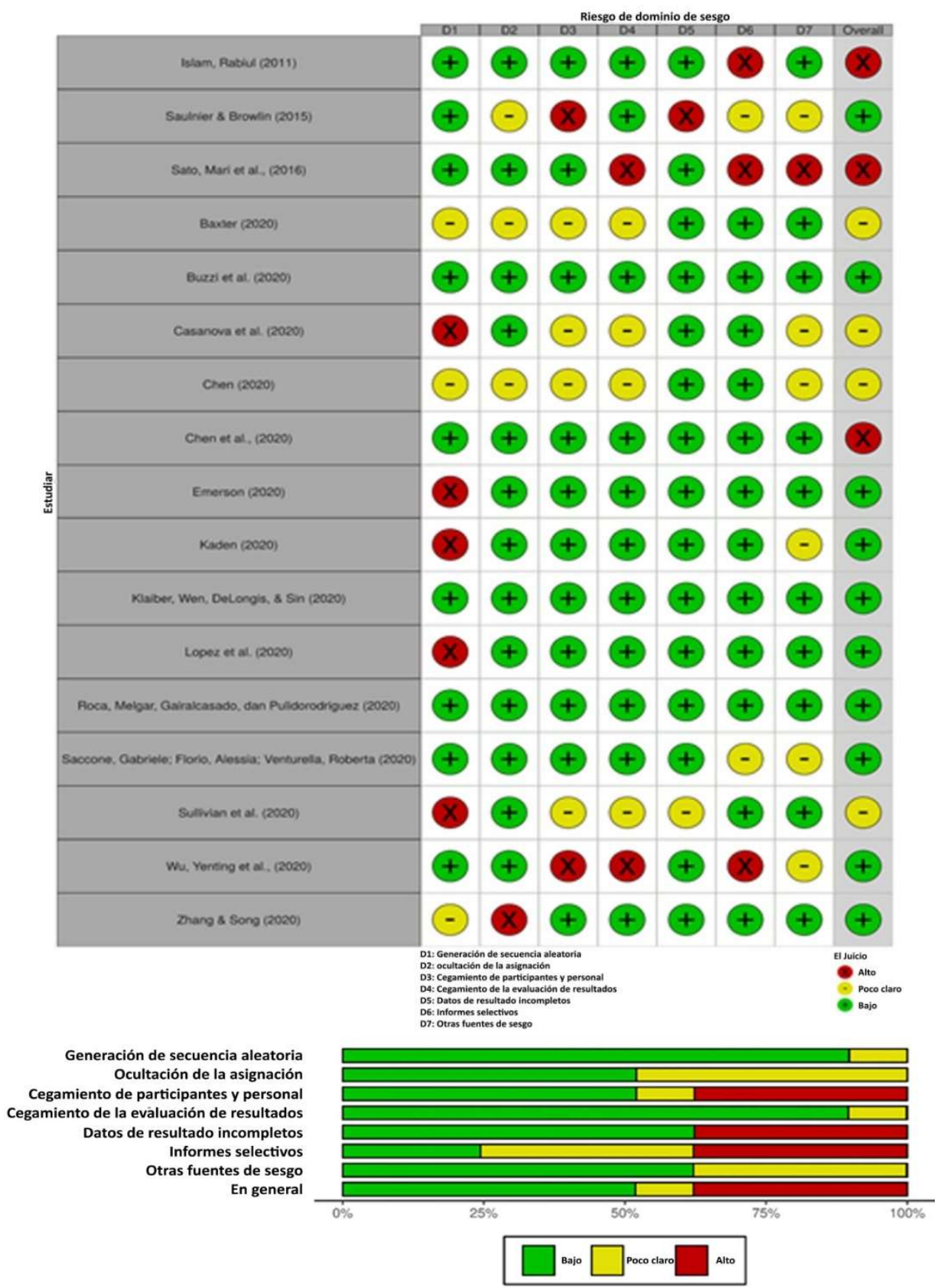




\section{DISCUSIÓN}

El contexto de afrontamiento en una situación de desastre, crisis o epidemia es una tendencia y técnica utilizada por los individuos para desarrollar los resultados que esperan de las condiciones o eventos. El afrontamiento se refiere a la capacidad de uno mismo para construir una estrategia o un conjunto de actividades para que las personas sobrevivan, se recuperen de situaciones e incluso desarrollen sus condiciones después de experimentar una crisis, un desastre o un brote ${ }^{(12,13)}$. El afrontamiento es una respuesta adaptativa a los factores estresantes, incluida la conducta de afrontamiento activa, como el proceso de pensamiento para encontrar soluciones, planificar los esfuerzos para resolver problemas / estresores percibidos. El afrontamiento como mecanismo de defensa mental para cada individuo también tiene condiciones de afrontamiento ineficaces, como los tipos de afrontamiento disfuncionales. Este afrontamiento muestra conductas de evitación, rechazo de la realidad, distracciones y conductas autodestructivas, incluso consumo de alcohol y narcóticos, y conductas emocionales maliciosas como la autoinculpación ${ }^{(13)}$.

Durante la pandemia, la aplicación del distanciamiento social provocó que las poblaciones vulnerables se convirtieran en uno de los grupos que experimentaban los peores impactos, por lo que se modificó el enfoque de las políticas en las personas vulnerables al enfrentar obstáculos durante la política de distanciamiento social. Su bienestar necesita atención debido al escaso acceso a los servicios sociales y de salud a los que pueden llegar los grupos vulnerables, especialmente los niños, las mujeres embarazadas y los adultos mayores ${ }^{(14)}$.

\section{Poblaciones Infantiles}

Durante la pandemia, los niños se ven obligados a adaptarse al entorno de forma consciente o no. A continuación, se muestran las formas de adaptación para ayudar a los niños que experimentan una pandemia:

- Niños enfermos: durante una pandemia, los niños con signos y síntomas de neumonía se clasifican como pacientes sospechosos de tener un riesgo positivo de COVID-19. Varios otros cambios relacionados con los protocolos de salud, como el uso de máscaras, mantener la distancia, prohibir abrazos, besos, jugar con otros pacientes o no permitir que las personas visiten a los niños durante la hospitalización. Las necesidades de atención de las enfermeras que se encuentran en servicio para este caso son fundamentales para reducir la transmisión entre adultos y niños.

- Niños sanos: los niños sanos que se quedan en casa durante mucho tiempo sin duda se enfrentarán al aburrimiento. Los padres tendrán más tiempo para jugar con sus niños pequeños. La creatividad de los padres para hacer que los niños se sientan como en casa es muy necesaria en este caso. Los padres que trabajan desde casa y los padres que no trabajan tendrán dificultades para tratar con sus hijos, especialmente después de la escuela. Los padres se ven obligados a adaptarse y a esforzarse por no quedarse atrás durante los métodos de aprendizaje de la escuela en línea. Los docentes y las escuelas también tienen un papel fundamental porque deben proporcionar un sistema y facilitar a los niños y padres para que las escuelas sigan funcionando de acuerdo con las competencias que deben alcanzarse. 
- Niños con necesidades especiales (discapacidades, cáncer, enfermedades crónicas y trastornos mentales): los niños con necesidades especiales necesitan esfuerzos, educación y adaptación más significativos y más dinámicos a las condiciones cambiantes durante una pandemia. Las medidas deben estar en consonancia con políticas de apoyo para que, con requisitos especiales, se sigan cumpliendo y no provoquen un empeoramiento de las condiciones.

Hacer frente a las barreras de los niños, enfermos o sanos, o niños con necesidades especiales, requiere una amplia atención de los padres. El obstáculo para los niños enfermos durante el período de cuidado requiere una atención especial, especialmente en los problemas psicológicos que tienden a ser mayores ${ }^{(14)}$. Esto se debe a que no todos los hospitales permiten que los niños que son positivos para COVID-19 estén acompañados por sus padres, mientras que también existe la posibilidad de que los niños sean separados de los padres que están positivamente enfermos y puestos en cuarentena. Necesita comprensión del entorno sobre las condiciones que enfrentan y la ayuda de otras personas, excepto los padres en cuyos hijos confiaron. Kazzaz ${ }^{(15)}$ declaró que la preparación hospitalaria y el enfoque en la prevención de infecciones y la atención a los procedimientos con aerosol deben considerarse en niños de alto riesgo mientras están en el hospital ${ }^{(15)}$.

Para los propios niños sanos, especialmente los niños que todavía tienen un calendario de vacunación básico, siempre deben administrarse vacunas. Durante el cierre de la escuela, los padres deben guiarlos y un maestro también debe acompañar a los padres. Kaden ${ }^{(16)}$ afirma que los profesores también tienen problemas para enseñar a los estudiantes, principalmente debido a la no disponibilidad de Internet en algunos de los hogares de los estudiantes en las regiones. Debido a la tecnología de Internet, los propios profesores encontrarán un desafío para explicar las materias, especialmente durante las prácticas, porque los profesores no pueden dirigirlas directamente(16). Roca, Melgar, Gairal-casado, \& Pulido-Rodriguez ${ }^{(17)}$ afirmaron que en el modelo de aprendizaje utilizado durante la pandemia, los niños necesitan mentores y ayuda para realizar sus trabajos; es necesario un entorno de apoyo mutuo entre los maestros, la escuela, la familia y la comunidad ${ }^{(17)}$.

Los niños con cáncer deben seguir recibiendo el tratamiento, pero con modificaciones para evitar la transmisión cruzada entre pacientes, especialmente con pacientes adultos. Los padres de niños con cáncer son los que más temen al riesgo de que sus hijos se infecten. Por lo tanto, los trabajadores de la salud pueden brindar planificación médica por teléfono o teleconferencia. Los niños que necesitan farmacoterapia oral pueden recibirla a través de los servicios de parto sin tener que ir al hospital(18). Los niños con retraso mental, discapacidad intelectual y autismo deben tener acceso a información de fácil comprensión ${ }^{(15)}$.

\section{Poblaciones de mujeres embarazadas}

Este estudio de revisión sistemática explora los efectos psicológicos de las crisis en las mujeres embarazadas. El embarazo afecta la fuerza mental, la salud física y, a veces, puede aumentar la susceptibilidad a la depresión. Un estudio muestra que los efectos psicológicos de las mujeres embarazadas sobre la pandemia de COVID-19 se encuentran en el rango de moderados a graves. La ansiedad se asocia principalmente con la transmisión de enfermedades; incluso los estudios muestran que en el primer trimestre tienen mayor ansiedad y problemas psicológicos que en el último 
trimestre(19). Son propensos a tener problemas de salud durante y después de las condiciones de crisis debido al estrés fisiológico y psicológico, el trauma y el acceso limitado a los servicios de salud. La exposición a condiciones de desastre o crisis es extensa y se convierte en un determinante importante de su salud fetal ${ }^{(20)}$. Además, su manera de afrontar las crisis, incluidos los brotes de enfermedades, es subjetivo y es muy probable que esté influido por la intensidad de los factores estresantes que sienten ${ }^{(13)}$.

El afrontamiento de las mujeres embarazadas con un alto nivel de estrés está relacionado con las condiciones de la crisis o los brotes de enfermedades que experimentaron ${ }^{(12,21)}$. Los estudios demuestran que tienen que hacer frente a niveles de estrés leves o moderados que se centran en la resolución de problemas (afrontamiento centrado en el problema). Además, el afrontamiento adaptativo, que a menudo utilizan en relación con condiciones de crisis, desastres naturales y epidemias, es un afrontamiento centrado en las emociones, es decir, actitudes y comportamientos positivos, incluida la búsqueda de apoyo emocional de otros y encontrar el lado positivo de los eventos. Los estudios muestran que este afrontamiento a menudo se demuestra cuando se enfrenta un desastre, una epidemia o una crisis prolongada más que un problema, un afrontamiento enfocado y un afrontamiento disfuncional(13).

Las políticas relacionadas con la cuarentena sanitaria o las restricciones sociales en la pandemia de COVID-19 también se han convertido en desafíos en los mecanismos de afrontamiento con el tipo de afrontamiento centrado en las emociones debido a las interacciones sociales limitadas ${ }^{(22)}$. Sin embargo, el uso de tecnología moderna y redes sociales puede superar esta condición. La comunicación intensiva con un número limitado de personas aún puede ayudar a expresar empatía, escuchar activamente, compartir información y lograr un afrontamiento emocional incluso con las restricciones sociales ${ }^{(22,23)}$. La ansiedad y depresión perinatal en mujeres embarazadas, que probablemente aumenten debido a crisis, desastres o epidemias, pueden tratarse con diversas actividades. Hacer pasatiempos o juegos puede mejorar las condiciones mentales; otros escuchan música, cantan, tocan instrumentos musicales y buscan información relacionada con los esfuerzos para mantener la salud. El apego entre los individuos y las crisis que enfrentan directamente (adaptarse a las condiciones de la pandemia) es un mecanismo de afrontamiento para mejorar el bienestar psicológico y reducir el estrés postraumático ${ }^{(20,22,24)}$.

\section{Poblaciones de adultos mayores}

COVID-19 ha traído cambios en las actividades de rutina diaria, los servicios de salud, la provisión de apoyo social, las habilidades sociales y las percepciones de los adultos mayores. Los grupos de adultos mayores enfrentan desafíos por pasar más tiempo en casa, contacto limitado con familiares, amigos y el medio ambiente, limitaciones para realizar trabajos y pasatiempos, y aumento de la ansiedad y el miedo a la muerte que puede ocurrir debido a una pandemia ${ }^{(25)}$. Con base en las Directrices de la Década del Envejecimiento Saludable 2020-2030 establecidas por la WHO, todos los países deben esforzarse al máximo en brindar servicios de salud completos para los adultos mayores a través de varias acciones, incluida la creación o el aumento de programas nacionales para concienciar sobre su bienestar y envejecimiento saludable, desarrollando políticas basadas en evidencias en todos los sectores de la sociedad 
para incrementar las capacidades de la comunidad, y aumentando el conocimiento y la conciencia pública, así como estimulando el diálogo entre generaciones ${ }^{(7)}$.

Los adultos mayores son el agregado del grupo más vulnerable durante la fase COVID-19. En general, experimentan barreras físicas, mentales y de perspectiva social y pueden convertirse en una pesada carga para los servicios de atención ${ }^{(26)}$. Los estudios informaron que este grupo tiene menos sentimientos de depresión y puede ser más resistente y tolerante emocionalmente que los del grupo más joven ${ }^{(27)}$. Aunque el nivel de aceptación y resiliencia impacta positivamente en la mejora de las condiciones de salud física, cognitiva y mental durante la fase pandémica, el nivel de resiliencia experimentado por ellos a menudo empeora debido a las limitaciones físicas de las condiciones crónicas. Los estados con un bajo nivel de arena y condiciones comórbidas pueden hacerlos más vulnerables que aquellos sin condiciones comórbidas. La tasa de curación para los adultos mayores depende en gran medida de la aceptación y la fortaleza de la comunidad. A continuación, se muestran algunos de los cambios que ocurren en los adultos mayores y formas de controlar los cambios que ocurren:

- Actividades diarias y apoyo comunitario: durante la fase pandémica, las personas que viven solas corren el riesgo de disminuir el apoyo a la salud de sus familias (como la atención de enfermedades, el control de la salud y el mantenimiento de la higiene del hogar). Sus actividades diarias se encuentran entre las más desafiadas durante la pandemia. La comunidad puede ayudarlos a satisfacer sus necesidades diarias durante una pandemia.

- Acceso a la información y educación pública: Quienes tienen menos probabilidades de acceder a los medios basados en Internet pueden ser facilitados a través de los servicios de medios tradicionales a los que tienen más probabilidades de acceder, como la televisión, los periódicos, las revistas y la radio.

- Atención de la salud: el envejecimiento se ha convertido en el factor de riesgo más importante para la aparición de enfermedades crónicas, incluidas las enfermedades cardiovasculares, la diabetes mellitus, el cáncer y las enfermedades cognitivas. Estas enfermedades se han vuelto comórbidas en adultos mayores, lo que los hace más susceptibles a otras enfermedades infecciosas. Los trabajadores de la salud deben minimizar su presencia física en los servicios de salud por el riesgo de transmisión. Como resultado, el riesgo de que tengan obstáculos para acceder a los servicios de salud es alto; por lo tanto, los trabajadores de la salud deben prestar especial atención al brindar servicios de salud de rutina a través de varias formas, como la telemedicina, la prescripción de medicamentos a largo plazo y las visitas domiciliarias regulares. Especialmente para la implementación de las visitas domiciliarias, los trabajadores de la salud deben estar al tanto de la transmisión de enfermeras que realizan visitas domiciliarias, que es la causa más común de transmisión de COVID19 que ocurre en adultos mayores.

- Problemas de salud mental de los adultos mayores: los adultos mayores que viven solos tienen un mayor riesgo de soledad y sentimientos de impotencia, y la pandemia actual puede exacerbar estas condiciones. Los trabajadores de la salud deben prestar atención a su bienestar psicológico como seguimiento del acceso limitado a Internet y a la información que se produce durante una pandemia. 
Los resultados mostraron que el $36 \%$ de los adultos mayores reportaron problemas de estrés, mientras que el $42.5 \%$ experimentó soledad, casi un tercio de ellos dijo la creciente soledad durante la fase de distanciamiento social. En general, los cambios en la actividad física, el estilo de vida y los patrones de sueño son significativos durante la fase de distanciamiento social. Estos hallazgos pueden convertirse en la base para implementar las políticas de los adultos mayores durante una pandemia(28). La pandemia ha cambiado la calidad de vida debido a las condiciones sociodemográficas y al acceso a los servicios de salud. Los adultos mayores que tienen una buena percepción de la salud, funciones familiares adaptativas, altos niveles de resiliencia y alto nivel de aceptación tienen un alto nivel de afrontamiento durante la pandemia. Los trabajadores de la salud deben prestar atención a los recursos individuales que pueden afectar su bienestar psicológico durante la fase pandémica para evitar el empeoramiento de sus condiciones de salud(29).

Los adultos mayores a menudo experimentan malas condiciones debido a la pandemia. Esto se ve agravado por la marginación de las políticas por parte del Gobierno. Durante la pandemia, los servicios de salud a menudo se niegan a que los grupos de adultos mayores se sometan a exámenes de detección porque tienen un alto riesgo de transmisión debido a su edad y pueden aumentar la incidencia de transmisión. Como resultado de la negativa de los servicios de salud, las familias (especialmente las esposas / hijas) a menudo se convierten en las únicas cuidadoras de ellos en el hogar. Esto también hace que las mujeres cuidadoras experimenten cambios en su trabajo y salud( ${ }^{(30)}$.

La pandemia provocó la aparición de nuevos servicios para adultos mayores que utilizan tecnología de telecomunicaciones y programas de atención domiciliaria. Hay una aceptación y una determinación cada vez mayores de mantener el afrontamiento positivo como las claves principales de este nuevo programa de servicios. Las amargas experiencias de la pandemia harán que el mundo piense más en cómo manejar la salud de los adultos mayores a través de nuevos enfoques ${ }^{(21)}$.

\section{CONCLUSIONES}

Una pandemia que ocurre durante un período prolongado puede aumentar la vulnerabilidad, especialmente en el sector de la salud, afectando a las poblaciones vulnerables de la sociedad, que consisten en niños, mujeres embarazadas y adultos mayores. Las poblaciones vulnerables tienen varios mecanismos de supervivencia y se ven afectadas significativamente por la presencia o ausencia de apoyo social de la familia, la comunidad y el gobierno. La atención del gobierno a la calidad de los servicios de salud específicos para poblaciones vulnerables puede mejorar su bienestar y estado de salud.

\section{REFERENCIAS}

1. World Health Organization. Coronavirus disease (COVID-19) pandemic. 2020.

2. Gugus Tugas Percepatan Penanganan Covid. Peta Sebaran Covid 19 di Indonesia. https://covid19.go.id/peta-sebaran. 2020.

3. Muhyiddin. Covid-19, New Normal dan Perencanaan Pembangunan di Indonesia. Indones J Dev Plan. 2020;4(2):240-52.

4. Takieddine $\mathrm{H}$, Tabbah $\mathrm{S}$ Al. Coronavirus pandemic: coping with the 
psychological outcomes, mental changes and the "New Normal" during and after COVID 19. Open J Depress Anxiety. 2020;(June):7-19.

5. Haleem A, Javaid M, Vaishya R. Effects of COVID 19 pandemic in daily life. Curr Med Res Pract. Elsevier Ltd; 2020;(April):10-2.

6. Norwegian Institute of Public Health. Social and economic vulnerable groups during COVID 19 pandemic: A rapid review. Nor Inst Public Heal. 2020;

7. WHO. Covid-19 Strategy Update. WHO Emergencies. 2020;(April).

8. Taylor S, Landry CA, Paluszek MM, Fergus TA, Mckay D, Asmundson GJG. COVID stress syndrome: Concept, structure, and correlates. Depress Anxiety. 2020;37:706-14.

9. Holuka C, Merz MP, Fernandes SB, Charalambous EG, Seal S V, Grova N, et al. The COVID-19 pandemic: Does our early life environment, life trajectory and socioeconomic status determine disease susceptibility and severity? Int J Mol Sci. 2020;21(5094):1-21.

10. Naga BSHB, Al-atiyyat NMH. The relationship between pain experience and roy adaptation model: Application of theoretical framework. Middle East $\mathrm{J}$ Nurs. 2014;8(1):18-23.

11. Hatami F, Hojjati H. Effect of Roy's adaptation model on the care burden of mothers of children under chemotherapy (a quasi-experimental study). Med -Surgical Nurs J. 2019;8(1):1-7.

12. Islam R. Vulnerability and coping strategies of women in disaster: A study on coastal areas of Bangladesh. Arts Fac J. 2011;

13. Chen T, Laplante DP, Elgbeili G, Brunet A, Simcock G, Kildea S, et al. Coping during pregnancy following exposure to a natural disaster: The QF2011 Queensland Flood Study. J Affect Disord. Elsevier B.V.; 2020;273(March):341-9.

14. Pradana AA, Casman, Nur'aini. Pengaruh kebijakan social distancing pada wabah COVID-19 terhadap kelompok rentan di Indonesia. J Kebijak Kesehat Indones. 2020;09(02):61-7.

15. Kazzaz YM, Alkhalaf H, Alharbi M, Shaalan M Al, Almuneef M, Alshehri A, et al. Hospital preparedness and management of pediatric population during COVID-19 outbreak. Ann Thorac Med. 2020;15(3):107-17.

16. Kaden U. COVID-19 school closure-related changes to the professional life of a K-12 teacher. Educ Sci. 2020;10(165):1-13.

17. Roca E, Melgar P, Gairal-casado R, Pulido-rodriguez MA. Schools that 'open doors' to prevent child abuse in confinement by COVID-19. Sustainability. 2020;12(4685):1-17.

18. Sullivan M, Bouffet E, Rodriguez-Galindo C, Luna-fineman S, Khan MS, Kearns $P$, et al. The COVID-19 pandemic: A rapid global response for children with cancer from SIOP, COG, SIOP-E, SIOP-PODC, IPSO, PROS, CCI, and St Jude Global. Pediatr Blood Cancer. 2020;(e28409):1-12.

19. Saccone G, Florio A, Venturella R. Psychological impact of coronavirus disease 2019 in pregnant women. Am J Obstet Gynecol. 2020;293-5.

20. Saulnier DD, Brolin K. A systematic review of the health effects of prenatal exposure to disaster. Int J Public Health. Springer Basel; 2015;

21. Chen L. Older adults and COVID-19 pandemic: Resilience matters. Arch Gerontol Geriatr. Elsevier; 2020;89(May):104124.

22. Polizzi C, Lynn SJ, Perry A. Stress and Coping in the Time of COVID-19: Pathways to Resilience and Recovery. Clin Neuropsychiatry. 2020;(April).

23. Sato M, Nakamura Y, F A, Horiguchi R, Tamaki R, Yoshizawa $T$, et al. Immediate needs and concerns among pregnant women during and after typhoon haiyan (Yolanda). Plos Curr Disaster. 2016;1. 
24. Wu Y, Zhang C, Liu H, Duan C, Li C, Fan J, et al. Perinatal depressive and anxiety symptoms of pregnant women during the coronavirus disease 2019 outbreak in China. Am J Obstet Gynecol. Elsevier Inc.; 2020;1-9.

25. World Health Organization. Decade of Healthy Ageing 2020-2030. 2020.

26. Zhang Q, Song W. The challenges of the COVID-19 pandemic: Approaches for the elderly and those with Alzheimer' s disease. Med Community. 2020;1(March):6973.

27. Klaiber P, Wen JH, DeLongis A, Sin NL. The Ups and Downs of Daily Life During COVID-19: Age Differences in Affect, Stress, and Positive Events. J Gerontol Soc Am. 2020;

28. Emerson KG. Coping with being cooped up : Social distancing during COVID-19 among $60+$ in the United States. Pan Am J Public Heal. 2020;44:1-7.

29. Lopez J, Perez-Rojo G, Noriega C, Carretero I, Velasco C, Martinez-Huertas J., et al. PSYCHOLOGICAL WELL-BEING AMONG OLDER ADULTS DURING THE COVID-19 OUTBREAK: A COMPARATIVE STUDY OF THE YOUNG-OLD AND THE OLD-OLD ADULTS. Int Psychogeriatrics. 2020;

30. Baxter LF. A Hitchhiker 's Guide to caring for an older person before and during coronavirus-19. Gend Work Organ. 2020;(April):1-11. 